\title{
Aspectos psicológicos em usuários de prótese ocular
}

\author{
Psychological aspects in ocular prosthesis users
}

\author{
Nara Lúcia Poli Botelho' \\ Marcos Volpini ${ }^{2}$ \\ Eurípedes da Mota Moura ${ }^{3}$
}

\section{RESUMO}

Objetivo: Avaliar os aspectos psicológicos decorrentes da anoftalmia unilateral adquirida, a luta pela recuperação estética com o uso de prótese ocular, assim como os fatores interpessoais envolvidos e a reintegração psicossocial destes pacientes. Método: Trinta pacientes portadores de anoftalmia unilateral adquirida, sem outras deformidades órbito-palpebrais e usuários de prótese ocular, sendo 16 do sexo masculino e 14 feminino, com idade variando de 12 a 66 anos, idade média 31,6 anos foram submetidos a exame oftalmológico e avaliação psicológica, por meio de questionário padronizado e semidirigido de 66 questões, com duração média de 2 horas no período de janeiro/2000 a março/2001. Resultados: A maior incidência de anoftalmia adquirida (47\%) está na faixa etária de 0 a 6 anos. Setenta por cento dos pacientes estão em processo de elaboração da perda $(n=21)$ e os mecanismos de defesa mais utilizados em face da situação instalada foram racionalização, repressão, negação e deslocamento. Trinta por cento aceitam a realidade atual. Após o evento desencadeador (ED) $37 \%$ manifestaram estado depressivo, tendo desaparecido os sintomas em $64 \%$ deles. Sessenta por cento adaptaram a prótese ocular (PO) logo após o tratamento cirúrgico ao passo que $40 \%$ adaptaram-na 2 ou mais anos após o ED. Esteticamente $70 \%$ estão satisfeitos ao passo que $30 \%$ estão insatisfeitos. Cinqüenta e três por cento revelam auto-estima rebaixada e 37\% auto-imagem distorcida. Conclusão: A integração entre os vários elementos da equipe multidisciplinar constituída por cirurgiões, protéticos, psicólogos e o apoio da família é fundamental durante todo o processo de luto instalado pela perda. Atitude positiva para com a pessoa acometida facilita a vivência da perda em sua totalidade, o que proporcionará a reestruturação dos aspectos interpessoais e reintegração psicossocial da pessoa, pois os recursos de enfrentamento delas estarão fortalecidos. A prótese exerce dupla função, pois de um lado devolve ao paciente sua autoimagem e auto-estima, possibilitando sua reintegração psicossocial, enquanto por outro lado, pode tornar-se um instrumento que afasta a possibilidade da perda ser vivenciada em sua totalidade, contribuindo para que esses indivíduos adotem atitudes de repressão diante da realidade instalada. Não há relação direta entre o resultado estético obtido com o grau de satisfação do paciente. O preconceito é outro fator importante, pois pode interferir ou não na reabilitação do sujeito, dependendo do comportamento que apresente diante da sociedade.
O trabalho foi realizado na Solótica

${ }^{1}$ Psicóloga do Departamento de Oftalmologia do Setor de Órbita da Santa Casa de Misericórdia de São Paulo e da Universidade Federal de São Paulo (UNIFESP).

${ }^{2}$ Médico Oftalmologista do Hospital Sírio Libanês. Responsável pelo Setor de Plástica Ocular da Santa Casa de Misericórdia de Santo Amaro - SP.

${ }^{3}$ Médico Assistente, Doutor e Chefe do Setor de Plástica Ocular da Clínica Oftalmológica do Hospital das Clínicas da Faculdade de Medicina da Universidade de São Paulo (USP).

Endereço para correspondência: Rua Jacques Félix, 76/121 - São Paulo (SP) CEP 04509-000

E-mail: narapolibotelho@hotmail.com

Recebido para publicação em 06.05.2002

Aceito para publicação em 23.01.2003
Descritores: Olho artificial/psicologia; Implantes orbitários/psicologia; Anoftalmia; Enucleação ocular; Preconceito; Adulto; Adolescente; Masculino; Feminino 


\section{INTRODUCÃO}

"A imagem que têm de si mesmos é tão confusa e vaga que nem sequer parecem saber quem são, o que são nem quais suas aspirações. Essa confusão se estende à sua atuação na vida. Sem se aterem dos fatos e às circunstâncias externas de sua vida, flutuam entre os sentimentos de inferioridade e de superioridade, entre considerarem-se fortes ou fracos, membros de um ou outro grupo, ou então seres totalmente isolados"(1).

O homem em suas primeiras manifestações artísticas dava pouca importância à reprodução do órgão visual, o que ficou evidenciado nas pinturas rupestres encontradas nas cavernas européias de Altamira e Lascaux. A história comprova que o interesse pela reprodução dos olhos cresceu paralelamente com o desenvolvimento das artes plásticas ${ }^{(2)}$.

Os antigos egípcios com a preocupação de embelezar suas estátuas confeccionavam olhos artificiais em ouro e pedras preciosas o que provavelmente suscitou a experimentação no homem $^{(2)}$.

Ambroise-Paré (1510-1590) idealizou um aro metálico que contornava a cabeça terminando em uma peça oval convexa que se adaptava à região orbital e pintada, simulava a região óculopalpebral. As primeiras próteses cavitárias eram muito rudimentares causando pressão no fundo da cavidade anoftálmica com conseqüente edema palpebral, irritação, infecção e complicações advindas do mau posicionamento das pálpebras ${ }^{(2)}$.

Em 1579, o vidro foi utilizado pela primeira vez para confeccionar próteses, proporcionando boa tolerância a este material. A Alemanha, desde 1835, foi o centro de produção dos melhores olhos artificiais. Ludwig Muller-Uri nesta época foi o primeiro a fabricar um olho artificial para um ser humano a pedido de um médico da cidade de Meiningem, que ficara extasiado com sua habilidade em confeccionar olhos de boneca. $\mathrm{O}$ resultado estético foi tão surpreendente que este trabalho altamente especializado, passou a ser uma tradição familiar conservada até hoje por seus descendentes que ainda produzem próteses em uma clínica de protéticos na cidade de Weesbaden ${ }^{(2)}$.

A partir da II Guerra Mundial a resina acrílica, material utilizado em próteses odontológicas, começou a substituir o cristal na confecção de olhos artificiais. Nesta época houve grande demanda devido à legião de mutilados provenientes do conflito que necessitavam de recuperação estética. Os esforços voltados para a técnica de confecção de próteses oculares, contribuem para os indivíduos que delas necessitam, em vários e complexos aspectos que a perda da visão e a mutilação do órgão implicam ${ }^{(2)}$.

Existe na vida de um indivíduo a tendência natural em manter todos os seus aspectos em constante estado de equilíbrio, como se fosse uma rede. A alteração em qualquer um desses aspectos pode eventualmente desarticular temporária ou permanentemente a vida do indivíduo e dependendo de como isto ocorre, pode haver conseqüências de maior ou menor gravidade ${ }^{(3)}$.

A visão é um dos órgãos do sentido que também atua nesse equilíbrio sendo responsável, em parte, por vários aspectos importantes para a sobrevivência do homem e de sua espécie. Defesa, segurança, alimentação, entre outros, são fatores ligados ao desenvolvimento do indivíduo. Assim, a perda da visão provoca marcantes alterações, deixando o homem mais exposto ao perigo, diminuindo sua capacidade de $\operatorname{adaptação~}^{(3)}$.

Há perdas, que por suas características, têm a tendência de provocar grandes alterações nas vidas das pessoas ${ }^{(3)}$. A perda de um olho é uma amputação, ocasionando um luto como a morte. Além do problema óbvio da cosmética final, existe uma consideração na qual, para algumas pessoas, é intolerável - a imagem corporal defeituosa sendo uma deformidade facial considerada muito maior que qualquer outra ${ }^{(4)}$.

O presente estudo tem por objetivo avaliar as condições sócio-emocionais desencadeadas pela anoftalmia unilateral adquirida, a luta pela recuperação estética com o uso de prótese ocular, assim como fatores interpessoais envolvidos na reintegração social destes pacientes.

\section{MÉTODOS}

Trinta (30) pacientes portadores de anoftalmia unilateral adquirida, sem outras deformidades orbito-palpebrais e usuários de prótese ocular, sendo 16 do sexo masculino e 14 do sexo feminino, com idades variando de 12 a 66 anos, idade média 31,6 anos foram submetidos a exames oftalmológicos de rotina (exame externo, avaliação da motilidade extrínseca, fundoscopia, refratometria, biomicroscopia e tonometria) e avaliação psicológica, através de um questionário padronizado e semidirigido com liberdade para qualquer resposta - com duração média de 2 horas, no período de janeiro/2000 a março/2001.

O questionário foi dividido em duas partes além da identificação pessoal do paciente, estética e constelação familiar. A primeira parte consistia de dados referentes à história natural, diagnóstico, idade do paciente quando ocorreu o evento desencadeador (ED), tempo decorrido para adaptação da prótese ocular e estética (a resposta ao item estética foi dada pela $1^{\text {a }}$ autora deste estudo, quem inclusive conduziu a avaliação psicológica). A segunda parte consistia da avaliação de aspectos psicológicos advindos da mutilação e seu reflexo intrapessoal bem como sua relação sócio-familiar. (Anexo 1)

\section{RESULTADOS}

O gráfico I mostra a idade dos pacientes na ocorrência do ED. Para fins didáticos as idades foram divididas de acordo com o desenvolvimento humano. Assim, 47\% da amostra tiveram o ED durante a infância pré-escolar (0-6 anos), 13\% foram acometidos na infância escolar (7-11 anos), 20\% na adolescência (12-20 anos) e 20\% em idade adulta (acima 21 anos).

Setenta por cento dos pacientes analisados $(n=21)$ estão em processo de elaboração da perda ${ }^{*}$, utilizando distintos

\footnotetext{
* Processo psíquico em que o paciente está compreendendo a situação de perda instalada, para mais tarde aceitá-la ou não ${ }^{(5)}$
} 


\begin{tabular}{|c|c|c|c|c|c|c|c|c|}
\hline $\mathbf{N}^{\circ}$ & Pacientes & Idade & Sexo & Profissão & Diagnóstico & E D & Estética & Idade $1^{\mathrm{a}} \mathrm{PO}$ \\
\hline 1 & A.C.S. & 18 & $\mathrm{M}$ & Estudante & Retinoblastoma & 2 anos & Boa & 3 anos \\
\hline 2 & F.F. & 15 & M & Estudante & Retinoblastoma & 1 ano & Boa & 2 anos \\
\hline 3 & G.A.S & 21 & $\mathrm{~F}$ & Monitora & Trauma - FP(AD) & 7 anos & Boa & 19 anos \\
\hline 4 & M.L.C. & 49 & $\mathrm{~F}$ & Professora: Piano & Uveíte & 5 anos & Boa & 5 anos \\
\hline 5 & N.D.F.C.M. & 24 & $\mathrm{~F}$ & Do lar & Uveíte & 1,5 ano & Razoável & 20 anos \\
\hline 6 & O.M. & 30 & M & Lavador & Trauma (AD) & 16 anos & Sem & 16 anos \\
\hline 7 & C.A.D.S. & 16 & $\mathrm{~F}$ & Estudante & Trauma - AVM & 16 anos & Razoável & 16 anos \\
\hline 8 & A.R.D.S. & 41 & M & Vendedor & Trauma - AVM & 33 anos & Boa & 33 anos \\
\hline 9 & F.R.I. & 24 & M & Estudante & Trauma - FAF (AT) & 20 anos & Boa & 22 anos \\
\hline 10 & C.A.N.P. & 45 & $\mathrm{~F}$ & Biomédica & Trauma - FA (AD) & 11 anos & Boa & 11 anos \\
\hline 11 & J.C.M.A. & 66 & M & Advogado & Trauma (AT) & 44 anos & Razoável & 47 anos \\
\hline 12 & W.M.A. & 57 & $\mathrm{~F}$ & Secretária & Uveíte & 9 anos & Razoável & 20 anos \\
\hline 13 & A.D.S.M. & 59 & M & Economista & Trauma - FP (AD) & 2 anos & Boa & 6 anos \\
\hline 14 & V.F.C.S. & 37 & $\mathrm{~F}$ & Professora & Trauma - AVM & 21 anos & Boa & 22 anos \\
\hline 15 & A.L.D.O.C. & 23 & M & Professor: Inglês & Trauma - FP (AD) & 2 anos & Boa & 2 anos \\
\hline 16 & P.N.I.P. & 26 & M & Militar reformado & Trauma - FP (AT) & 26 anos & Boa & 26 anos \\
\hline 17 & R.R.R. & 42 & $\mathrm{~F}$ & Professora: Português & Trauma - FP (AD) & 3 anos & Boa & 14 anos \\
\hline 18 & A.A.S. & 46 & M & Empresário & Trauma - AVM & 24 anos & Boa & 24 anos \\
\hline 19 & N.L.P.B. & 22 & $\mathrm{~F}$ & Psicóloga & Herpes zóster & 5 anos & Boa & 19 anos \\
\hline 20 & P.L.W. & 14 & $\mathrm{~F}$ & Estudante & Retinoblastoma & 3,5 anos & Ruim & 4 anos \\
\hline 21 & R.D.M. & 20 & M & Ajudante Varejão & Trauma - AVM & 19 anos & Boa & 22 anos \\
\hline 22 & A.J.S.J. & 30 & M & Metalúrgico & Úlcera & 1 ano & Boa & 22 anos \\
\hline 23 & F.J.F. & 43 & M & Bancário & Trauma - FP (AD) & 6 anos & Boa & 7 anos \\
\hline 24 & J.S.D.L. & 12 & $\mathrm{~F}$ & Estudante & Retinoblastoma & 7 meses & Razoável & 4 anos \\
\hline 25 & A.J.L.D.S. & 29 & M & Estudante & Trauma - FA (AD) & 7 anos & Boa & 14 anos \\
\hline 26 & A.S.D.S. & 28 & $\mathrm{~F}$ & Jornaleira & Glaucoma & 0 anos & Razoável & 2 anos \\
\hline 27 & J.D.S.C. & 22 & $\mathrm{~F}$ & Desempregada & Trauma - FP (AD) & 8 meses & Ruim & 7 anos \\
\hline 28 & C.S.D.M. & 36 & $\mathrm{~F}$ & Dentista & Herpes ocular & 21 anos & Boa & 21 anos \\
\hline 29 & J.E.S.F. & 18 & M & Estudante & Trauma - FP (AT) & 16 anos & Sem & 18 anos \\
\hline 30 & F.D.B.M. & 34 & M & Mâitre & Trauma (AD) & 20 anos & Boa & 22 anos \\
\hline
\end{tabular}

mecanismos de defesa*: $29 \%$ defendem-se por meio da repressão ${ }^{* *}, 14 \%$ através de deslocamento ${ }^{* * *}, 19 \%$ através de negação ${ }^{* * * *} \mathrm{e}$ os $38 \%$ restantes através de racionalização ${ }^{* * * * *}$. Assim, apenas $30 \%$ dos pacientes aceitam a realidade atual (Gráfico II e III).

Trinta e sete por cento dos pacientes manifestaram transtornos de humor após o ED, compreendido como um estado depressivo. Desses, 64\% superaram os sintomas depressivos enquanto os $36 \%$ restantes continuam atuando do mesmo modo (Gráfico IV e V).

Observou-se que $60 \%$ dos pacientes adaptaram a prótese ocular imediatamente após o tratamento cirúrgico enquanto $40 \%$ adaptaram dois ou mais anos após o ED (Gráfico VI).

Diversos tipos de processos psíquicos inconscientes, cuja finalidade consiste em afastar um evento gerador de angústia da percepção consciente ${ }^{(5)}$.

** Mecanismo de defesa que impede pensamentos dolorosos atingirem a consciência ${ }^{(5)}$.

*** Mecanismo de defesa que desloca, transfere ou canaliza sentimentos, em geral agressivos, em pessoas, objetos ou situações menos ameaçadoras ${ }^{(5)}$.

**** Mecanismo de defesa que não permite a consciência perceber aspectos que seriam dolorosos para as pessoas ${ }^{(5)}$.

***** Mecanismo de defesa que abstrai vivências afetivas sob premissas lógicas, justificando as atitudes das pessoas ${ }^{(5)}$.

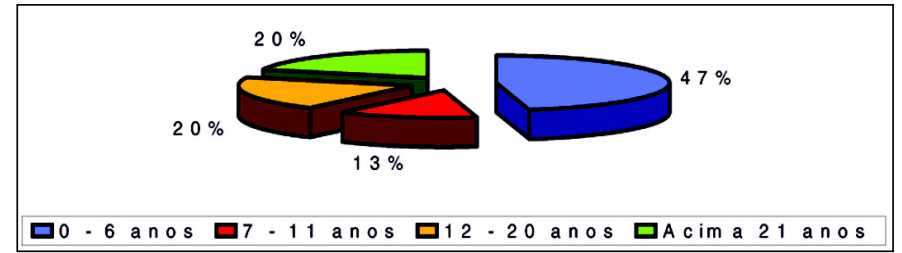

Gráfico I - Idade da ocorrência da anoftalmia

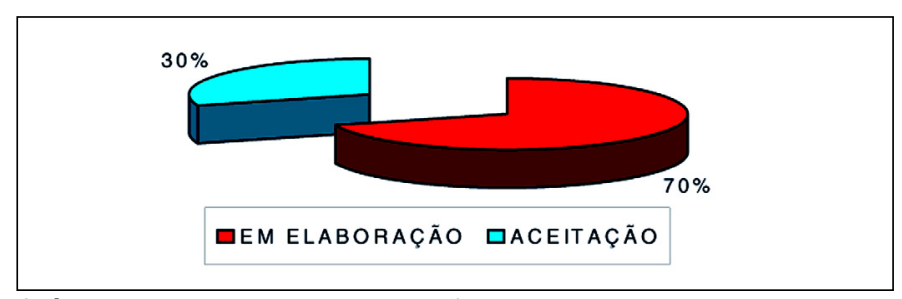

Gráfico II - Processo de elaboração da perda

Observou-se que $70 \%$ da amostra revelam contentamento estético, embora $30 \%$, encontram-se insatisfeitos esteticamente com a mesma (Gráfico VII). 


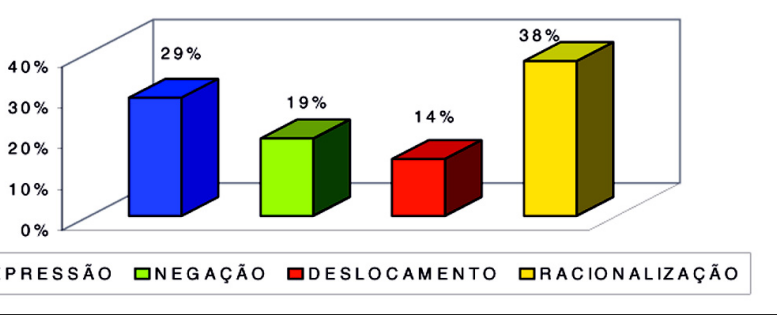

Gráfico III - Mecanismos de defesa

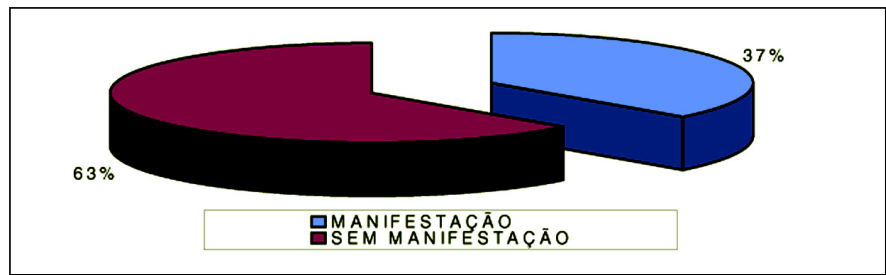

Gráfico IV - Estado depressivo após ED

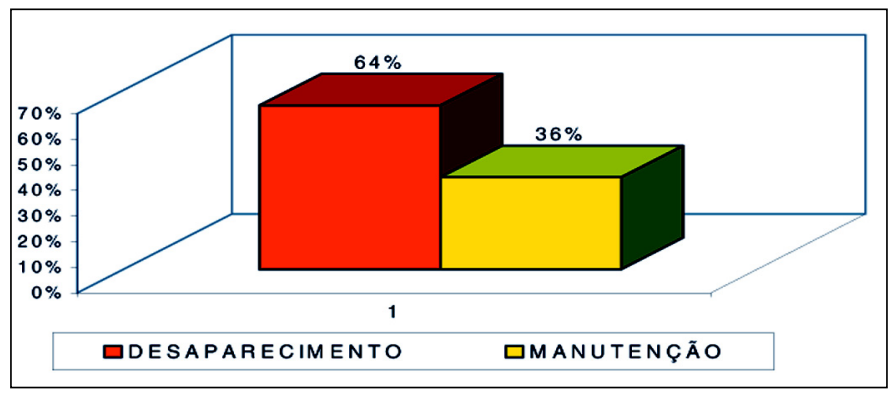

Gráfico V - Estado depressivo na época da entrevista

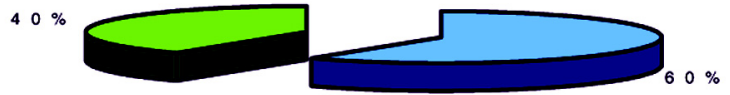

口A P Ó S T R A T A M E N T O C IR Ú R G IC O 口ANOS APOS O EVENTO

Gráfico VI - Período da adaptação da prótese ocular

Verificou-se que $80 \%$ da amostra atribuem à adaptação da prótese ocular um ganho secundário enquanto $20 \%$ valorizam somente a perda da visão e suas implicações reais.

Trinta e três por cento omitem a falta do bulbo ocular para terceiros.

Cinqüenta e sete por cento revelam uma auto-estima* rebaixada e $37 \%$ auto-imagem ${ }^{* *}$ distorcida - dados associados às questões 43, 44, 45 e 46. (Gráfico VIII e IX).

Observou-se que $13 \%$ da amostra atribuíram à orientação médica, técnica, psicológica ou religiosa, prestada na ocasião do ED uma influência positiva durante a realização do tratamento.

Verificou-se que $3 \%$ dos pacientes desenvolveram dependência alcoólica após o ED.

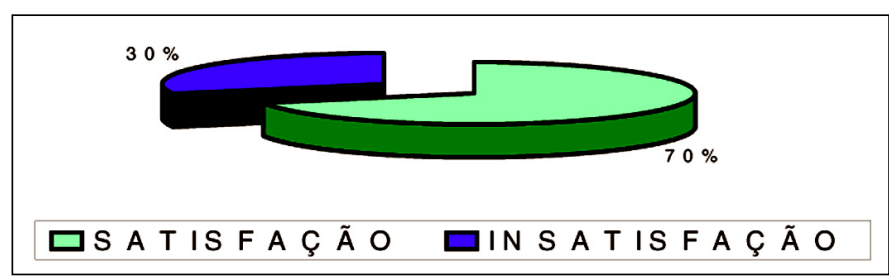

Gráfico VII - Conceito estético

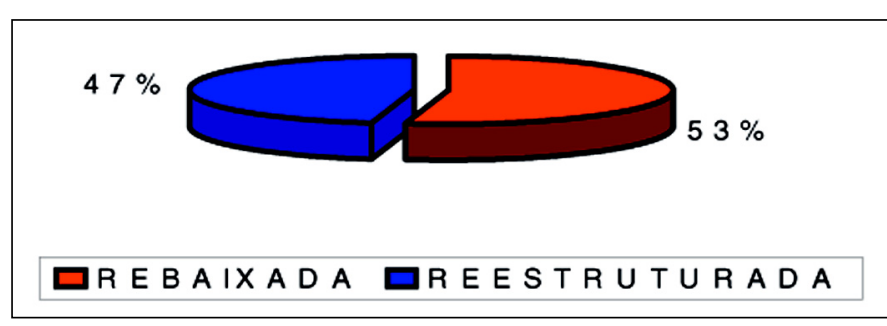

Gráfico VIII - Auto-estima

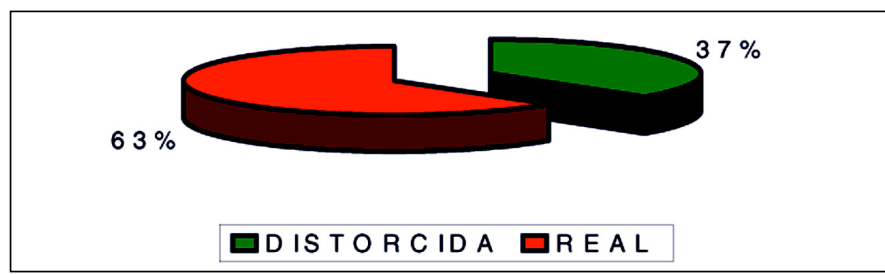

Gráfico IX - Auto-imagem

Treze por cento dos pacientes atribuíram à família expectativas que não foram supridas, levando-os a criar fantasias persecutórias, geralmente relacionadas à figura materna e $33 \%$ atribuem culpa pela perda que foram acometidos, projetando na mesma a incapacidade de atender suas próprias necessidades. De acordo com os dados obtidos $37 \%$ dos pacientes demonstram que foram superprotegidos por familiares enquanto $63 \%$ demonstram que apenas receberam cuidados básicos após o ED.

Verificou-se que $70 \%$ da amostra acreditam ter o comportamento alterado em relação a atitudes frente à vida, crenças, valores e moral após o ED, enquanto $30 \%$ permanecem agindo do mesmo modo que agiam antes. Trinta e sete por cento dos pacientes afirmaram que o comportamento dos outros para com eles sofreu mudanças após o ED, em relação ao modo como passaram a ser tratados e $63 \%$ dizem não terem notado diferença.

\section{DISCUSSÃO}

A prótese ocular é um instrumento de reabilitação de quatro aspectos distintos: anatômico, estético, pessoal e interpessoal. Segundo os aspectos anatômico e estético, a PO

\footnotetext{
* Sentimento de importância ou valor que cada pessoa tem em relação a si mesma ${ }^{(6)}$. ** Concepção corpórea que cada pessoa tem de $\mathrm{si}^{(6)}$.
} 


\begin{tabular}{|ccc|}
\hline \multicolumn{2}{|c|}{ Tabela 6. Idade e estado civil dos pacientes satisfeitos com o } \\
tratamento \\
Idade & Estado Civil \\
$\mathbf{N}^{\circ}$ & 18 anos & Solteiro \\
2 & 15 anos & Solteiro \\
3 & 21 anos & Solteiro \\
4 & 49 anos & Solteiro \\
5 & 24 anos & Solteira \\
7 & 16 anos & Solteira \\
9 & 24 anos & Solteiro \\
10 & 45 anos & Solteiro \\
11 & 66 anos & Casado \\
12 & 57 anos & Viúva \\
13 & 59 anos & Casado \\
14 & 37 anos & Casada \\
15 & 23 anos & Solteiro \\
17 & 42 anos & Casado \\
19 & 22 anos & Solteiro \\
20 & 14 anos & Solteiro \\
21 & 20 anos & Solteiro \\
22 & 30 anos & Casado \\
23 & 43 anos & Casado \\
29 & 18 anos & Solteiro \\
30 & 34 anos & Solteiro \\
\hline
\end{tabular}

\begin{tabular}{|rrc|}
\hline \multicolumn{3}{|c|}{$\begin{array}{c}\text { Tabela 7. Pacientes que aceitam a realidade em relação ao tempo } \\
\text { decorrido entre o ED e a adaptação da PO }\end{array}$} \\
$\mathbf{N}^{\circ}$ & Ocorrência ED & Adaptação Po \\
6 & 16 anos & 16 anos \\
9 & 20 anos & 22 anos \\
11 & 44 anos & 47 anos \\
15 & 2 anos & 2 anos \\
16 & 26 anos & 26 anos \\
19 & 5 anos & 19 anos \\
28 & 21 anos & 21 anos \\
29 & 16 anos & 18 anos \\
30 & 20 anos & 22 anos \\
\hline
\end{tabular}

A pessoa que é acometida na infância por uma deficiência visual, terá mais chances de reintegrar-se totalmente à vida do que aquele que sofre um corte abrupto das suas atividades em outra fase do desenvolvimento, já que terá que re-significar suas cognições dali em diante. No presente estudo observouse que durante a infância a aceitação da deficiência é maior, devido ao convívio precoce com as limitações desencadeadas pela falta da visão e sua integração no processo de modificações naturais do desenvolvimento.

A perda instalada durante a adolescência e idade adulta dificulta o processo de elaboração da nova realidade, pois há uma compreensão total da dimensão da realidade e a tendência, muitas vezes, de significá-la como o fim de uma vida normal, sem nenhuma manifestação de enfrentamento dessa realidade.

Quando a deficiência é instalada a pessoa torna-se fragilizada, todos os seus recursos internos são mobilizados, levando-a atuar no mundo conforme suas experiências prévias,

$\begin{array}{|cc|}\begin{array}{c}\text { Tabela 8. Estética da PO dos pacientes que elaboraram a perda } \\ \mathbf{N}^{\circ}\end{array} & \text { Estética PO } \\ 7 & \text { Razoável } \\ 9 & \text { Boa } \\ 11 & \text { Razoável } \\ 15 & \text { Boa } \\ 16 & \text { Boa } \\ 19 & \text { Boa } \\ 28 & \text { Boa } \\ 29 & \text { Sem PO } \\ 30 & \text { Boa } \\ \text { As respostas referentes à questão "O que você acha da Prótese?" fizeram } \\ \text { menção ao conforto estético atribuído à peça e não à beleza dela ou semelhança } \\ \text { com o olho contralateral }\end{array}$

recursos de enfrentamento, mecanismos de defesa e percepção. Todo o seu funcionamento é influenciado e, portanto, deve ser compreendido e respeitado dentro de suas possibilidades e limitações, já que todas as pessoas trarão para o problema instalado sua personalidade ${ }^{(5)}$.

O processo de elaboração da perda corresponde ao período em que o indivíduo encontra-se em um estado de lamentações pela vida, exigindo esperança de quem o está vivenciando. Viver a situação de perda em sua totalidade proporciona ao indivíduo recursos internos para aceitar a realidade atual e condições de reintegração à vida, já que a perda da visão e a conseqüente cegueira adquirida se aprofunda e prolonga quando se encoraja o paciente a fugir da aceitação da situação que pesa sobre ele ${ }^{(7)}$.

Os mecanismos de defesa são inerentes ao processo de perda. Identificou-se a negação como um processo que evita a consciência perceber uma dor insuportável. A repressão como um bloqueio para a consciência de pensamentos dolorosos advindos da situação instalada. O deslocamento como expressão da agressividade contida pela realidade sentida como irreversível e a racionalização como a abstração de vivências afetivas sobre premissas lógicas que tentam justificar o comportamento ${ }^{(5,8)}$.

A omissão da falta do bulbo ocular gera, na maioria das vezes, o medo de exclusão, repulsa, estigma ou ainda o sentimento de inferioridade. A união e interação dos indivíduos são baseadas no olhar mútuo e a interação de um olho com outro, termina no momento em que a direção da função é perdida. Assim, o desconforto pela diferença leva uma pessoa a olhar para o chão evitando o olhar do outro ${ }^{(9)}$.

Quanto à perda do órgão explicitamente, não houve prejuízo profissional, pois as pessoas não contam que não têm o globo ocular nessas situações. Quanto à estética, muitas vezes há prejuízo sim, principalmente quando o cargo a ser preenchido requer boa aparência. Outros fatores que podem influenciar as relações profissionais são: a crença que o empregador tem de que empregar um deficiente físico pode lhe trazer complicações no andamento da empresa, por esse precisar sair do serviço para acompanhamento médico constante ou simplesmente o preconceito de empregar um deficiente físico. 


\begin{tabular}{|c|c|c|c|c|c|c|c|c|c|c|}
\hline \multicolumn{11}{|c|}{ Tabela 9. Identificação da amostra } \\
\hline $\mathbf{N}^{\circ}$ & Idade & Sexo & $\begin{array}{l}\text { Natura- } \\
\text { lidade }\end{array}$ & Profissão & $\begin{array}{c}\text { Estado } \\
\text { civil }\end{array}$ & Filhos & ED & $\begin{array}{c}\text { Local } \\
\text { Tratamento }\end{array}$ & $\begin{array}{l}\text { Local } \\
\text { Adp. PO }\end{array}$ & Estética \\
\hline 1 & 18 & M & MG & Estudante & Solteiro & --- & Retinoblastoma & HBP & Solótica & Boa \\
\hline 2 & 15 & M & SP & Estudante & Solteiro & -- & Retinoblastoma & Clínica Particular & Solótica & Boa \\
\hline 3 & 21 & $\mathrm{~F}$ & SP & Monitora & Solteira & 2 & Trauma - FP (AD) & $\begin{array}{l}\text { Santa Casa } \\
\text { Sto. Amaro }\end{array}$ & Solótica & Boa \\
\hline 4 & 49 & $\mathrm{~F}$ & SP & Professora: Piano & Solteira & -- & Uveíte & Clínica Particular & Solótica & Boa \\
\hline 5 & 24 & $\mathrm{~F}$ & PR & Do lar & Casada & 3 & Uveíte & $\begin{array}{l}\text { Hospital de } \\
\text { Rubião Jr }\end{array}$ & Solótica & Razoável \\
\hline 6 & 30 & M & PR & Lavador & Solteiro & 1 & Trauma (AD) & Hospital Público & Solótica & $\mathrm{S} / \mathrm{PO}$ \\
\hline 7 & 16 & $\mathrm{~F}$ & SP & Estudante & Solteira & -- & Trauma - AVM & Clínica Particular & Solótica & Razoável \\
\hline 8 & 41 & $M$ & SP & Vendedor & Casado & 5 & Trauma - AVM & HSP & Solótica & Boa \\
\hline 9 & 24 & $M$ & SP & Estudante & Solteiro & -- & Trauma - FAF (AT) & Clínica Particular & Solótica & Boa \\
\hline 10 & 45 & $\mathrm{~F}$ & SP & Biomédica & Solteira & -- & Trauma - FA (AD) & $\begin{array}{l}\text { Hospital das } \\
\text { Clínicas }\end{array}$ & Solótica & Boa \\
\hline 11 & 66 & M & PE & Advogado & Casado & 3 & Trauma - (AT) & Clínica Particular & Solótica & Razoável \\
\hline 12 & 57 & $\mathrm{~F}$ & SP & Secretária & Viúva & 2 & Uveíte & Clínica Particular & Solótica & Razoável \\
\hline 13 & 59 & $\mathrm{M}$ & SP & Economista & Casado & 2 & Trauma - FP (AD) & Clínica Particular & Solótica & Boa \\
\hline 14 & 37 & $\mathrm{~F}$ & MG & Professora & Casada & 2 & Trauma - AVM & Clínica Particular & Pro-Ocular & Boa \\
\hline 15 & 23 & $M$ & MA & Professor: Inglês & Solteiro & - & Trauma - FP (AD) & Clínica Particular & Pro-Ocular & Boa \\
\hline 16 & 26 & M & RS & Militar reformado & Casado & 1 & Trauma- FP (AT) & Clínica Particular & Solótica & Boa \\
\hline 17 & 42 & $\mathrm{~F}$ & MG & Professora: Português & Casada & --- & Trauma - FP (AD) & Clínica Particular & Solótica & Boa \\
\hline 18 & 46 & $\mathrm{M}$ & SP & Empresário & Casado & 4 & Trauma - AVM & Clínica Particular & Solótica & Boa \\
\hline 19 & 22 & $\mathrm{~F}$ & SP & Psicóloga & Solteira & -- & Herpes zóster & Clínica Particular & Solótica & Boa \\
\hline 20 & 14 & $\mathrm{~F}$ & SP & Estudante & Solteira & -- & Retinoblastoma & Hospital Público & Solótica & Ruim \\
\hline 21 & 20 & $M$ & MG & Ajudante Varejão & Solteiro & -- & Trauma - AVM & $\begin{array}{l}\text { Hospital das } \\
\text { Clínicas }\end{array}$ & Solótica & Boa \\
\hline 22 & 30 & $\mathrm{M}$ & SP & Metalúrgico & Casado & 2 & Úlcera & $\begin{array}{l}\text { Santa Casa } \\
\text { Sto. Amaro }\end{array}$ & Solótica & Boa \\
\hline 23 & 43 & $M$ & SP & Bancário & Casado & 1 & Trauma - FP (AD) & Hospital Público & Solótica & Boa \\
\hline 24 & 12 & $\mathrm{~F}$ & MG & Estudante & Solteira & -- & Retinoblastoma & Hospital Público & Solótica & Razoável \\
\hline 25 & 29 & $\mathrm{M}$ & SP & Estudante & Solteira & --- & Trauma - FA (AD) & Hospital Público & Solótica & Boa \\
\hline 26 & 28 & $\mathrm{~F}$ & BA & Jornaleira & Casada & -- & Glaucoma & Hospital Público & Solótica & Razoável \\
\hline 27 & 22 & $\mathrm{~F}$ & SP & Desempregada & Solteira & --- & Trauma - FP (AD) & Hospital Público & Solótica & Ruim \\
\hline 28 & 36 & $\mathrm{~F}$ & RJ & Dentista & Casada & 2 & Herpes ocular & Hospital Particular & Pro-Ocular & Boa \\
\hline 29 & 18 & $\mathrm{M}$ & SP & Estudante & Solteiro & -- & Trauma - FP (AT) & Hospital Público & Pro-Ocular & $\mathrm{S} / \mathrm{PO}$ \\
\hline 30 & 34 & $\mathrm{M}$ & PR & Mâitre & Solteiro & --- & Trauma (AD) & Clínica Particular & Solótica & Boa \\
\hline
\end{tabular}

A depressão pode ser vivida como outra fase do processo de luto, quando um estado de profundo pesar e infinita tristeza pela situação de perda são instalados. Assim, todos os aspectos que envolvem a situação tendem ser sentidos como negativos, dificultando o processo de elaboração ${ }^{(10)}$.

Observou-se que os indivíduos apresentaram transtornos de humor após o ED, caracterizados por um estado depressivo desencadeado por uma condição médica. O estado depressivo manifesta-se a partir de um significado interno que cada pessoa faz da situação que está vivendo, o que determinará se a pessoa está ou é, deprimida. Apesar dessas alterações de humor terem características de um transtorno depressivo maior, o estado depressivo não se inclui nessa categoria por ser uma condição médica instalada previamente. Esse estado depressivo configura-se por: duração média de duas a quatro semanas, alteração no apetite, diminuição de energia, hipersonia, sentimentos de desvalia e até pensamentos recorrentes sobre morte, acompanhado por intenso sofrimento e comprometimento do desempenho profissional, atuação social e outras áreas importantes da vida do indivíduo ${ }^{(11)}$.
A família deve ser considerada dentro do contexto da deficiência, pois esta não atinge apenas o sujeito, mas também a todos os seus membros. A deficiência que acomete um membro de uma família pode desencadear desorganização e ruptura enquanto em outras pode haver reorganização e fortalecimento $^{(12)}$. Perdas são invariavelmente associadas a castigos por coisas que se fez ou deixou de fazer na vida, havendo necessidade de atribuir culpa a alguém. E a família, como se verificou, se sente culpada muitas vezes pela perda que o ente adquiriu, sentindo-se agredida pelo próprio paciente e impotente diante de seu sofrimento. Assim, superprotegem o familiar num acúmulo súbito de atenção, tentando perdoarem-se dessa situação, inibindo seu desenvolvimento e inferindo-lhe o sentimento de que é doente ${ }^{(8)}$.

A família ainda pode marginalizar o ente acometido, considerando-o frágil, deixando-o agir apenas por ordens e afastando-o da possibilidade de integração sócio-familiar ${ }^{(13)}$. Assim, a pessoa formará seu autoconceito negativamente em virtude do seu desenvolvimento ter sido comprometido, enquanto uma atitude familiar contrária possibilita um autoconceito po- 
sitivo, já que o relacionamento é revestido de autoconfiança, proporcionando auto-estima elevada

Quanto ao relacionamento com amigos, os pacientes tanto puderam perceber aqueles que se afastaram após o acontecimento, como os que se aproximaram ainda mais. Os pacientes relatam este último como sendo amigos de verdade, para todos os momentos, sejam estes alegres ou tristes. Vale ressaltar que o afastamento das pessoas remete muitas vezes, na percepção inconsciente da vulnerabilidade de cada um, já que todos estão sujeitos a situações da mesma ordem. Com o sexo oposto pode haver interferência quando a pessoa acometida apresenta uma concepção de si inferior aos demais, desenvolvendo sentimentos de desvalia ou mesmo apresentando uma imagem corpórea distorcida, o que dificulta estabelecer relações interpessoais afetiva-emocionais. O sexo, o estado civil e a faixa etária independe, o que determina dificuldade ou facilidade é conseqüência da história emocional de cada um.

Um tratamento mais abrangente além dos procedimentos técnicos e cirúrgicos, avaliando os aspectos psicossociais que circundam o contexto em que a perda da visão ocorre, possibilita aos pacientes melhores condições de convívio com a deficiência e suas conseqüências ${ }^{(14)}$. $\mathrm{O}$ aspecto social deve ser considerado, uma vez que a pessoa precisa de seu lugar na sociedade para reintegrar-se, inclusive como meio de sobrevivência. A resposta da sociedade será permeada pelo preconceito, inerente à tentativa de controle social. A cultura dita o modo de pensar e comportar apesar do livre-arbítrio, reforçando uma sociedade opressiva. O alvo, com medo da exclusão, repulsa e estigma adotará uma determinada forma de posicionamento perante os outros, reforçando ou não a continuidade do preconceito $^{(1)}$.

A participação da equipe multidisciplinar é indispensável para que o indivíduo se fortaleça na elaboração da perda. Com o auxílio de especialistas em cirurgia plástica ocular, protéticos e psicólogos pode se conseguir a reabilitação, pois somente após o paciente voltar a sentir, fazer e experimentar como antes é que se pode considerá-lo psicologicamente reabilitado ${ }^{(3,15)}$.

É possível associar ao apoio psicológico especializado uma perda melhor vivenciada e, portanto uma aceitação mais estruturada da nova realidade, mas que estatisticamente no estudo não foi significativa, uma vez que somente três por cento da amostra recebeu esse tipo de apoio.

\section{CONCLUSÃO}

Quando o ED ocorre em crianças (zero a 11 anos) a tendência à aceitação e elaboração da perda é maior do que em adolescentes e adultos devido ao convívio precoce com suas limitações. A adaptação da $\mathrm{PO}$ e a conseqüente recuperação da estética contribuem para os aspectos pessoal e interpessoal não sofrerem modificações naturais de seu desenvolvimento. $\mathrm{Na}$ fase adulta o ED provoca uma ruptura súbita na rotina de atividades e hábitos do indivíduo. A modificação estética insta- lada leva a uma distorção da auto-imagem e rebaixamento da auto-estima que pode estimular fantasias de exclusão social, aniquilamento perante o mercado de trabalho, impossibilidade de estabelecer vínculos afetivo-emocionais, contribuindo para que essas pessoas adotem uma atitude fatalista diante da situação, inviabilizando a reintegração social e dificultando o processo de elaboração da perda.

A PO é um instrumento de reabilitação de quatro aspectos distintos: anatômico, estético, pessoal e interpessoal.

A incidência de estados depressivos foi grande na população estudada, sendo a orientação médica, suporte psicológico e apoio familiar fundamentais para o alívio de sintomas e reintegração social.

A família contribui de modo positivo ou negativo na maneira de enfrentamento que o paciente adotará diante da perda. Atitude exagerada de superproteção ao indivíduo pode levá-lo a desencadear um sentimento de inferioridade, prejudicando a recuperação de sua auto-estima, o que dificulta sua reintegração. Conclui-se também que, muitas vezes, a atitude de proteção excessiva que a família deposita em seu familiar é um reflexo do sentimento de culpa que a mesma expressa pela perda que acometeu o paciente.

A grande maioria dos pacientes acredita ter o comportamento alterado a partir do ED devido ao amadurecimento pessoal, introjeção de novos valores morais e posicionamento mais ativo diante da vida, suscitados a partir da experiência que proporcionou um aprendizado positivo e adaptativo frente à nova realidade.

\section{ABSTRACT}

Purpose: To evaluate psychological disturbances caused by acquired unilateral anophthalmia, the struggle for aesthetic recuperation on use of the ocular prosthesis, as well as involved interpersonal factors and psychosocial reintegration of these patients. Method: Thirty (30) patients with acquired unilateral anophthalmia without any other deformities in the orbital region and users of ocular prosthesis (16 males, 14 females) aged between 12 and 66 years, mean age 31.6 years, were submitted to an ophthalmic examination and psychological evaluation through a standardized questionnaire focusing on 66 questions lasting two hours in the period from January, 2000 to March, 2001. Result: The highest incidence of acquired anophthalmia (47\%) was in the age ranging from 0 to 6 years old. Seventy $(70) \%$ of the patients are in the process of elaboration of their loss $(n=21)$. The most used mechanisms of defense in the current situation were rationalization, repression, denial and displacement. Thirty (30)\% accept the present situation. After the triggering event (TE) 36\% manifested a depressive state $64 \%$ of whom got over the symptoms of depression. Sixty percent $(60 \%)$ adapted the ocular prosthesis right after the surgical treatment while $40 \%$ adapted it two or 
more years after the TE. Aesthetically $70 \%$ are satisfied while $30 \%$ are unsatisfied. Fifty-three (53)\% show low self-steem, $37 \%$ distorted self-image. Conclusion: An integration among various elements of the multidisciplinary team made up of surgeons, ocularists, psychologists and support from family and friends is fundamental during the whole process of mourning due to the loss. A positive attitude towards the person dealing with the issue minimizes the loss in all of its aspects, offering recovery of interpersonal aspects and psychosocial reintegration as he/she is given adequate resources to become more secure. The prosthesis has two functions: on the one hand it returns to the patient self-image and self-steem allowing his/her exposure without discrimination, which allows his psychosocial reintegration. On the other hand it becomes an instrument, which removes the possibility of the loss to be lived in its totality, which contributes to behavior of repression of the patients instead of acceptance of the new reality mainly when the prosthesis is adapted after the TE. There is no direct relationship between the obtained aesthetic result and the degree of satisfaction of the patients. Prejudice is another important factor because it may interfere or not in the rehabilitation.

Keywords: Eye, artificial/psychology; Orbital implants/ psychology; Anophthalmos; Eye enucleation; Prejudice; Adult; Adolescent; Male; Female

\section{REFERÊNCIAS}

1. Crochík JL. Preconceito - Indivíduo e Cultura, $2^{\mathrm{a}}$ ed. São Paulo: Robe Editorial; 1997. p. 152 .

2. Fonseca EP, Rosé MCM. Histórico da prótese ocular. In: Fonseca EPD. Prótese ocular. São Paulo: CIP; 1987. p.17-22.

3. Cyrillo PI. Aspectos psicológicos relacionados aos portadores de lesões oculares e a utilização de prótese. In: Fonseca EPD. Prótese ocular. São Paulo: CIP; 1987. p.181-7.

4. Lubkin V. Psychological considerations. In: Stwart WB. Plastic surgery. California: San Francisco; 1984; p.42-4.

5. Rapport CR, Fiori WR, Davis C. Teorias do desenvolvimento. São Paulo: EPU; 1981.

6. Dicionário Aurélio da língua portuguesa. Rio de Janeiro: Editora Nova Fronteira; 1977.

7. Cholden L. Some psychiatric problems in the rehabilitation of the blind. Bull Manninger Clin 1854;18(3):1-28.

8. Kübler-Ross E. Sobre a morte e o morrer. São Paulo: Martins Fontes; 1996. p. 299.

9. Lubkin V. Enucleation. the hunt for verisimilitude. Adv Ophthalmic Plast Reconstr Surg 1988;7:255-62.

10. Lubkin V. The public face or psychological and legal complexities in plastic ophthalmic surgery. Br J Ophthalmol 1975;59:593-9.

11. DSM-IV - Manual diagnóstico e estatístico de transtornos mentais. Porto Alegre: Artes Médicas; 1995.

12. Kovács MJ. Deficiência adquirida e qualidade de vida - possibilidades de intervenção psicológica. In: Becker E, et al. Deficiência: alternativas de intervenção. São Paulo: Casa do Psicólogo; 1997. p.95-123.

13. Masini EFS. Intervenção educacional junto à pessoa deficiente visual. In: Becker E, et al. Deficiência: alternativas de intervenção. São Paulo: Casa do Psicólogo; 1997. p.73-92.

14. Petrowski DD. Care of an artificial eye after enucleation - with comments from the author's personal experience. J Ophthalmic Nurs Technol 1986; 5:135-9.

15. Van Doorne JM, van Waas MA, Bergsma J. Facial disfigurement after cancer resection: a problem with an extra dimension. J Invest Surg 1994;7:321-6. 


\section{Identificação}

Nome:

Data de nascimento:

Idade:

Sexo:

Naturalidade:

Escolaridade:

Profissão:

Estado Civil:

Filhos:

Endereço:

CEP:

Telefone:

Evento Desencadeador:

Estética:

Local de Tratamento / Adaptação da Prótese Ocular:

Constelação Familiar

\section{Parte A - História da Doença}

1. O que o tornou portador de prótese ocular?

2. Há quanto tempo isto aconteceu?

3. Quantos anos você tinha nessa época?

4. Sob quais circunstâncias ocorreu?

5. Qual foi a $1^{\mathrm{a}}$ atitude no momento em que ocorreu?

6. Que tipo de tratamento se submeteu?

7. Como o tratamento se desenvolveu?

8. Você se submeteu a cirurgias?

9. Por que?

10. As possibilidades de resultados the foram informadas?

11. Como?

12. Durante o tratamento suas dúvidas foram esclarecidas?

13. Quando a prótese foi sugerida?

14. Por quem?

15. Quanto tempo após a situação ter sido instalada?

16. Como foi sugerida?

17. Teve todas as explicações para o uso da prótese?

18. Quem cuida ou cuidava da assepsia?

19. Como foi aprender a fazer a assepsia?

\section{Parte B - Aspectos psicológicos Reflexos do Evento Desencadeador}

20. Como você se sentiu diante da idéia de usar uma prótese ocular?

21. Como foi o caminho para tornar-se portador da prótese ocular?

22. O que acha da prótese?

23. Como você encarou a situação no momento em que foi adaptada?

24. Como as outras pessoas encararam essa situação?

25. Como foi para sua mãe, seu pai e seus irmãos em relação à situação que se instalou?

26. Para seu cônjuge / companheiro(a) e filhos?

27. Vocês conversam sobre isso?

28. Todos sabem o que lhe aconteceu?

29. Como foi durante a fase escolar?

30. Como foi no trabalho?

31. Há algo que você sinta como marcante que ocorreu nessas fases?

32. Você acha que seu comportamento mudou quando a situação se instalou?

33. O que mudou?

34. Você acha que o comportamento dos outros em relação a você mudou?

35. O que mudou?

36. O que você pensa sobre tais mudanças?

37. Como você se sente diante dessas mudanças?

38. O que significa para você tais mudanças?

39. Você pensa que as mudanças se dão devido à sua atitude estabelecida para com os outros ou devido às atitudes dos outros para com você?

40. Quanto à sua vida cotidiana, há algo que você não faz mais ou com menor freqüência?

41. O quê?

42. Por qual motivo?

43. Como é para você a limitação diante de alguns aspectos?

44. Como você se vê sendo portador de uma lesão ocular e usuário de prótese?
45. Como você pensa que os outros o vêem?

46. E como você se sente por isso?

47. Você enfrenta ou já enfrentou problemas profissionais ou sociais por ter uma lesão ocular e ser usuário de prótese?

48. Você fala sobre isso com os outros?

49. Como é falar ou não sobre isso?

50. O que você pensa disso?

51. Como você se descreve antes e depois de se tornar portador de uma lesão ocular?

52. O que você pensa sobre isso?

53. O que você diz sobre o caminho que percorreu ou percorre para a reabilitação?

54. Houve em algum momento alguém ou algo que teve uma forte influência seja ela positiva ou negativa, em sua maneira de encarar a situação?

55. Como você se sente diante disso?

56. O que significa para você os cuidados que recebeu da família quando a situação foi instalada?

57. Em termos de vida, valores, moral, educação e sociedade, você sentiu alguma mudança devido à situação?

58. Suas crenças e valores se mantiveram, fortaleceram ou enfraqueceram?

59. Como você se sente ao falar sobre isso?

60. Você se sente feliz hoje?

61. Quais eram ou são suas expectativas sobre a prótese ocular?

62. Você faz algum tipo de tratamento psicológico?

63. Há quanto tempo?

64. O que você pensa da sociedade em que vive?

65. O que você pensa sobre o preconceito?

66. Você acha que o preconceito é uma questão cultural, criada pela sociedade ou uma situação criada pelo "diferente" no modo como se apresenta para essa sociedade? 